\title{
Other combinations than top quark mass measurements at the LHC
}

\author{
Mara Soares*t \\ CIEMAT, Madrid \\ mara.senghi@ciemat.es
}

In this contribution we summarize the on combinations of the top quark measurements at the LHC. Combinations of the top quark production cross sections measurements, both in pairs and in single final states, and measurements of the top quark properties are presented. The combinations of the top quark mass are presented in other contribution to this conference. These activities are carried out within the LHCTOPWG at CERN, a forum of discussions where theorists and experimentalists work together to exploit the full potention of top quark physics at the LHC.

8th International Workshop on Top Quark Physics, TOP2015

14-18 September, 2015

Ischia, Italy

\footnotetext{
*Speaker.

†n behalf of the ATLAS and CMS Collaborations.
} 


\section{Introduction}

Since the LHC started collecting data from pp collision, the physics of the top quark has been prioritary topic within the high-energy physics community. The urge for understanding the mechanism electroweak symmetry breaking, and its implications on the physics of the top quark, impulsed the LHC Collaborations to build a scientific program where the top quark is studied with a high level of scrutiny, while pursuing excellent precision.

The LHCTOP working group (LHCTOPWG) plays a key role on these two purposes: throughout the combinations of independent measurements, the methodologies and results of ATLAS and CMS Collaborations are thoroughly verified against each other. Better precision is directly achieved due to the statistical independency of the input measurements, and the differences in the detector, analysis methods, etc, bring out a complementarity on the systematic uncertainties of the measurements that may further increase the precision of the combined results.

Furthermore, the direct contact between experimentalists and theorists in forums of discussions promoted within the LHCTOPWG are beneficial for the entire community, since theory and experiment are constantly challenging and aiding each other towards reaching better precision. For instance, some of the most important sources of experimental systematic uncertainties come from the modeling of the $t \bar{t}$ system in Monte Carlo - thus, discussions with Monte Carlo experts and theorists on the best tunes and generation parameters can help improving the models, reducing systematic uncertainties. In turn, higher and higher experimental precision reveals some features in the data that require more accurate theoretical calculations to be described - the most recent case being the top quark $p_{\mathrm{T}}$ spectra observed by ATLAS and CMS Collaborations, for which a full NNLO calculation was presented for the first time in this conference [1].

This contribution is organized as follows. Section 2 gives a brief overview of the method used for the combinations. In the following section, a short summary of the ATLAS and CMS combinations of top quark production and properties measurements is given. Top quark mass combinations are discussed in another contribution to this conference [2]. In section 4 we conclude and present some prospects for the combinations measurements at 8 and $13 \mathrm{TeV}$ that could be of general interest.

\section{Combination method}

The combinations presented in this contribution make use of the Best Linear Unbiased Estimator (BLUE) [3] method. BLUE is a simple and robust method that provides an weighted sum of the input measurements, taking into account the input uncertainties and their correlations, in order to minimize the uncertainties on the combined results. So, the most challenging aspect of the combinations is to identify which sources of uncertainties of the input measurements are of similar nature, and to estimate the correlations between them.

Uncertainties derived from statistical limitations of the independent samples or those related to detector effects are easily mapped from one experiment to another, and are uncorrelated. Likewise, uncertainties coming from the same source and estimated in both experiments using similar tools, as for instance the proton parton density functions (PDF) uncertainty, can be easily identified as 
correlated inputs to the BLUE method. But for other uncertainties the situation is much more complicated.

In some cases, a quoted uncertainty is in fact a sum of uncertainties from several sources. Some of these components may be correlated, others uncorrelated, and some of them may even lack an equivalent on the other experiment. An example is the jet energy scale uncertainty, for which components from similar effects, for instance arising from particularities of the method and Monte Carlo modeling, are correlated, while others, such as the detector response to the jet reconstruction or of statistical components, are uncorrelated. These and other sources sum up to one single jet energy scale uncertainty for each experiment, requiring an extensive study on the correlations between them, as documented in Ref. [4].

Since the first combinations were performed in the LHCTOPWG, the understanding of the correlations between ATLAS and CMS is constantly improving. For this reason, the input uncertainty groups and their correlations, considered for different combinations, may differ across time. In any case, the approach adopted in all cases is that if a correlation is not well known at the time of a combination, the best knowledge existing at that moment is used, and the effect of this unknown input is studied by repeating the combination varying the assumed correlation in a large range around its central value. These stability checks are reported in all combinations presented in this contribution.

\section{Summary of the results}

The results listed in this section are representative of the working areas in the LHCTOPWG. Several of them are presented in more details in other talks in this conference. Only the most recent results from which working area are mentioned.

\subsection{Top quark pair cross section}

Top quark pair producion cross sections are excellent tests of the standard model. They can be compared to very accurate theory predictions, and used to constrain the existing limits on supersummetry. A large amount of measurements, covering almost all possible final states, was provided by ATLAS and CMS collaborations. Given that t⿱亠䒑 pairs are abundantly produced at the LHC, many of these measurements were performed at very early stages of the data taking period of Run I, using only a fraction of the now available data.

The most up-to-date LHC combinations at $7 \mathrm{TeV}$ correspond to a result published in 2012 [6] using ATLAS measurements in the di-lepton, lepton+jets and all-jets channels (datasets luminosities ranging from 0.7 to $1.02 \mathrm{fb}^{-1}$ ) and CMS measurements in the di-lepton, lepton+jets, $\mu \tau_{\text {had }}$ and all-jets channels $\left(0.8\right.$ to $\left.1.09 \mathrm{fb}^{-1}\right)$.

At $8 \mathrm{TeV}$ [5], the ATLAS "legacy" measurement in the e $\mu$ channel using the full dataset of $20.3 \mathrm{fb}^{-1}$ was combined with a CMS measurement based on $5.3 \mathrm{fb}^{-1}$ of data. Although the original CMS measurement used 3 di-lepton final states, only the $\mathrm{e} \mu$ channel was considered for the combination.

The results, summarized in Fig. 1, show a remarkable agreement with the standard model predictions at NNLO. The combinations improve the results accuracy in about 10\%, compared 
to the most precise input measurement. Systematic luminosity uncertainties drive the precision limations.
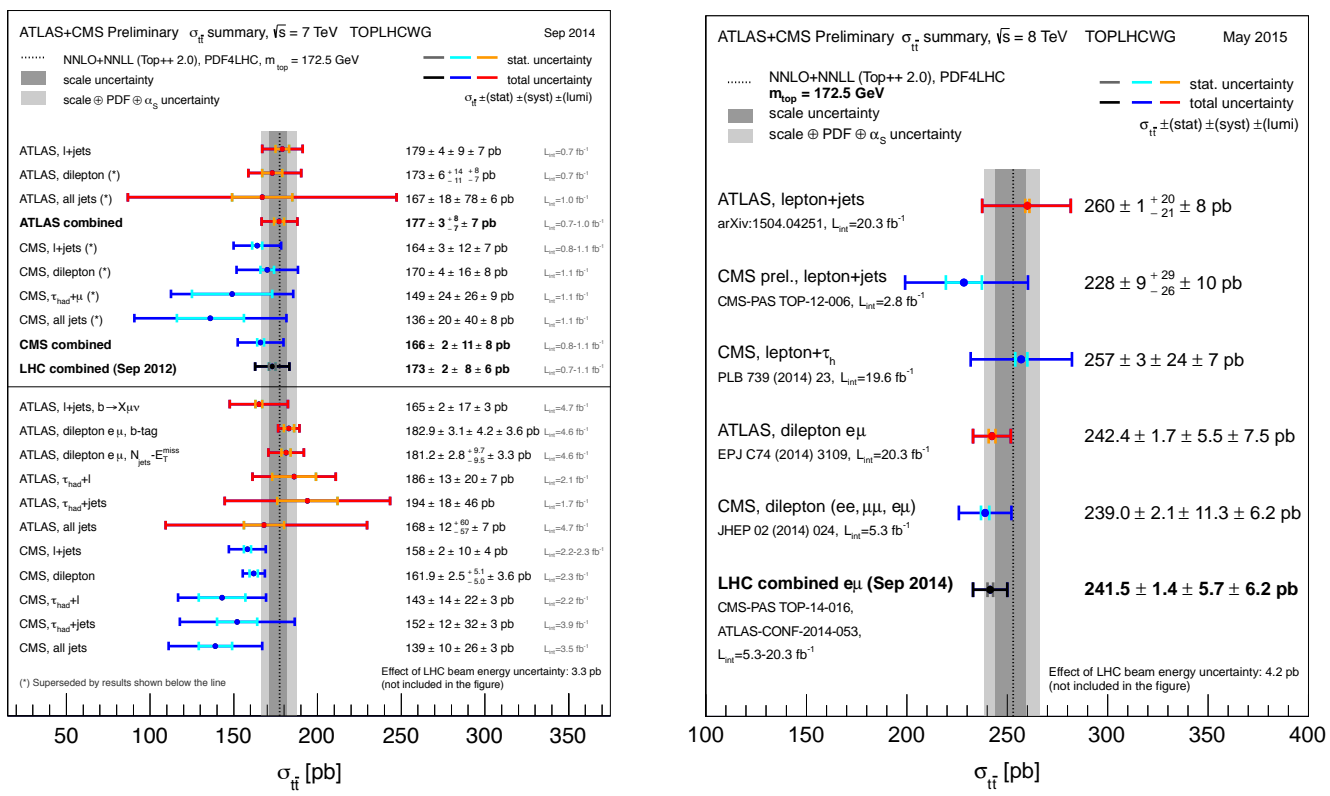

Figure 1: Summary of the ATLAS and CMS t⿱t $\bar{c}$ cross section measurements used in combinations, and combined results at $7 \mathrm{TeV}$ [5] (left) and $8 \mathrm{TeV}$ [6] (right).

\subsection{Single top quark cross section and CKM matrix element $V_{\mathrm{tb}}$}

The single top quark production in association with a $\mathrm{W}$ boson was measured by ATLAS and CMS using di-lepton final states. Both measurements used similar analysis strategies, with boosted decision trees technology to separate signal and background. The LHC combination [7] of these measurements reached a precision of $18.6 \%$, dominated by the theory modeling uncertainty. The most recent LHC combination of top quark production measurements in the $t$-channel [8] dates back to 2012, and utilized only about one forth of the full $8 \mathrm{TeV}$ dataset. An update of the LHC combination using the most recent input measurements is ongoing.

Assuming that the Wtb vertex is left-handed, and that both $V_{\mathrm{ts}}$ and $V_{\mathrm{td}}$ are negligible compared to $V_{\mathrm{tb}}$, the single top quark cross section via $V_{\mathrm{tb}}=\sigma_{\text {meas }} / \sigma_{\text {theory }}$. An uncertainty on the theoretical cross section $\sigma_{\text {theory }}$ due to the uncertainty on the proton beam energy was not considered in the original measurements, and was added for the combination. Although the gain in precision is not remarkable, this combination proves the compatibility of CMS and ATLAS results for this indirect measurement.

The combined values of the single top cross sections, and of the $V_{\mathrm{tb}}$ derived from it are shown in Fig. 2.

\subsection{Charge asymmetry in top quark decays}

An excess observed on the foward-backward asymmetry in dilepton final states from tit production at the Tevatron. At the LHC, the Tevatron asymmetry on the initial parton content is lost (since 

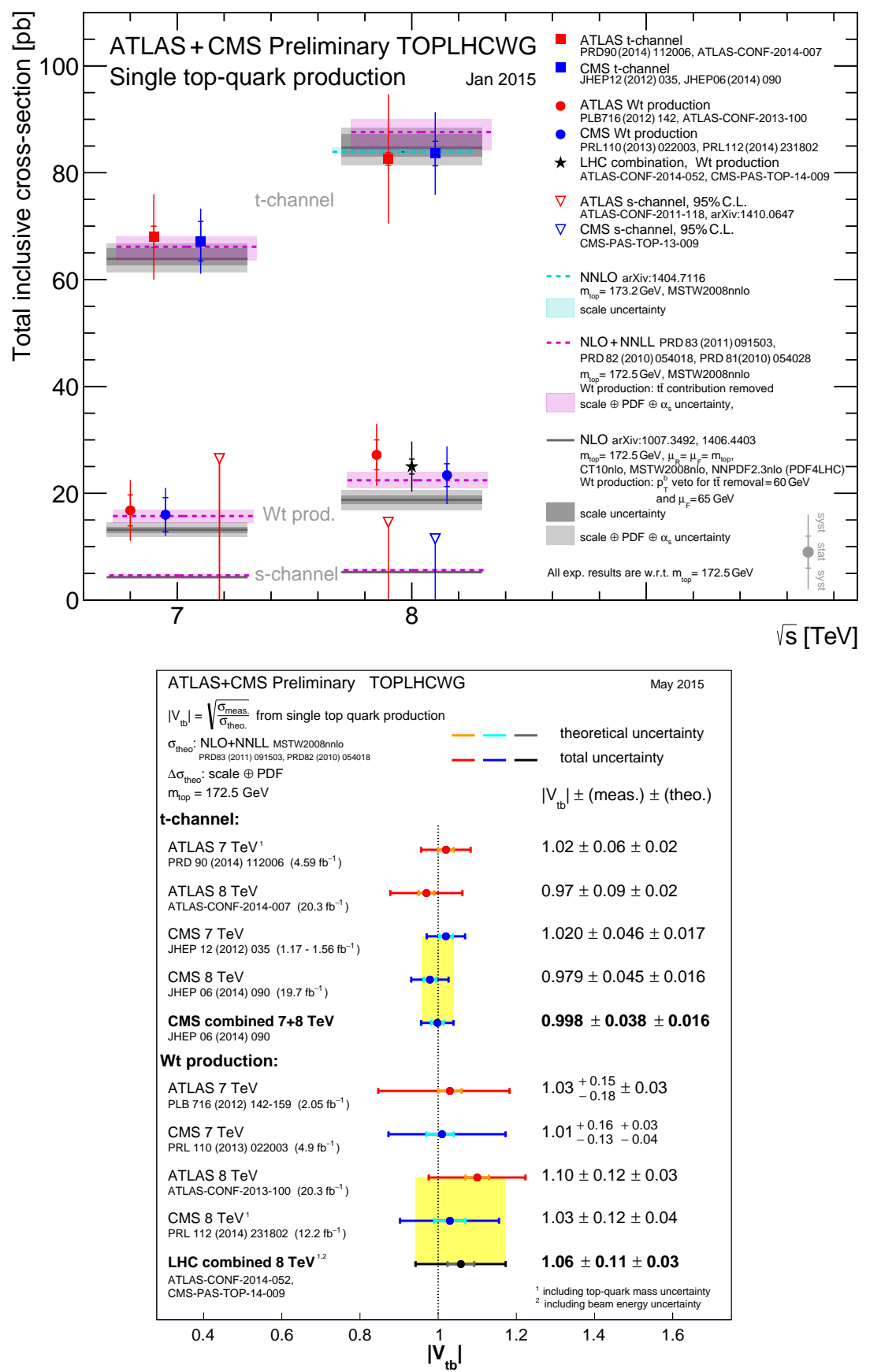

Figure 2: Summary of the ATLAS and CMS single top quark measurements, and combined results for $t$ and Wt channels: production cross sections (top) and $\left|V_{\mathrm{tb}}\right|$ (bottom).

the LHC collides pp instead of $p \bar{p}$ as Tevatron) and $\bar{t}$ production is dominated by gluon-gluon interaction, as opposed to dominant quark interactions at the Tevatron. That means, the very same asymmetry is not present at the LHC, instead only a much milder asymmetric effect is expected. That is: the top quarks rapidity distribution is slightly broader than the top antiquarks distribution, simply because on average, in pp collisions quarks (from valence and sea) carry more momentum 
than antiquarks (from sea distributions).

Still, the great interest in studying this small effect is to verify at the LHC any potential anomaly that could be related to an experimental result that is not perfectly described by the standard model predictions at the Tevatron. For this reason, the $t \bar{t}$ charge asymmetry measurements from the CMS and ATLAS Collaborations at $7 \mathrm{TeV}$ were combined [9]. In the original measurement, ATLAS used a fully Bayesian unfolding technique, with a marginalization procedure for the systematic uncertainties. For the combination, the uncertainties before the marginalization were used, so that each source of systematic uncertainty could be accessed.

The combination, shown in Fig. 3 (left), improves in 10\% the precision of the most accurate single measurement, and is still the most precise experimental result to this date. No anomaly was observed, since the experimental result is in good agreement with the standard model.

\subsection{W-boson polarization in top quark decays}

The W-boson polarization in top quark decays is of particular interest because they can be used to set limits on anomalous coupling on the Wtb vertex. The three polarization fractions (longitudinal, left and right) must sum up to unity, leaving two numbers to be measurement. These two fractions are measured from one single angular distribution of the top decay products and are, therefore correlated. This is an additional complication to the combination, since the inputs to the BLUE methods become larger correlations matrices. On the other hand the combined results may greatly improve the limits on anomalous couplings. The very first measurements from ATLAS and CMS were combined [10] yielding the results presented in Fig. 3 (right).
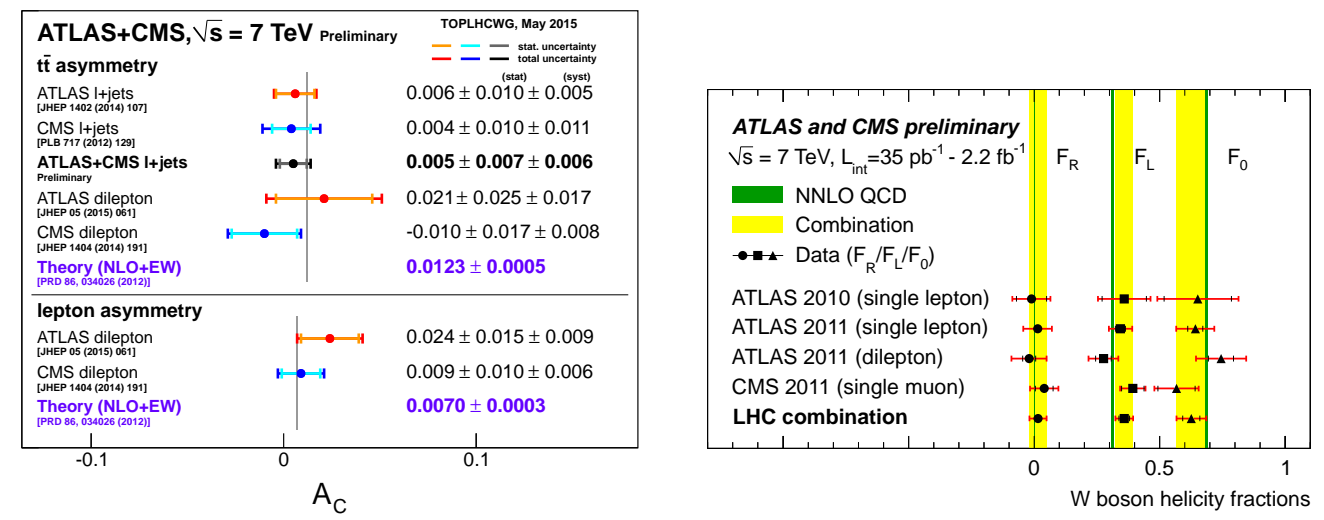

Figure 3: Summary of the top quark properties used in LHC Combinations: charge asymmetry [9] (left) and W boson helicity [10] (right).

\section{Prospects and conclusions}

Several measurements of the top quark performed by the ATLAS and CMS collaborations at 7 and $8 \mathrm{TeV}$ have been combined, such as top quark pairs and single top quark production, charge asymmetry and W-boson polarization in top-quark decays. Some of these combinations have been superseded in precision by more recent measurements from each experiment, while others are still the most precise to date. 
But far beyond the improvement on precision on a particular measurement, these combinations, and of the work related to them in the framework of the LHCTOPWG, resulted greatly beneficial for the advancements on the physics of the top quark in the last years. In the process of combining the measurements, the consistency of the results is checked across experiments, and the adopted methodologies are discussed. A much better understanding of the systematic uncertainties estimated independently by each collaboration, and the level of correlation between them, has been achieved. Better tools have been developed for the modeling of the top quark production. Potential inconsistencies in the tuning of Monte Carlo parameters are more rapidly spotted, either through direct comparisons between the two experiments or in discussions with theoreticians. Insights on the best way to present the data often arise in discussions, leading to more robust comparisons with models and data interpretation.

Currently, a rich program of activities is being carried out within the LHCTOPWG. In the following, a non-extensive list of the LHCTOPWG results expected to come in the near future is presented.

The ongoing combinations of the recently published Run I "legacy" measurements, for instance, top quark inclusive and boson-associated productions, lepton asymmetry, etc, are expected to provide the most accurate set of experimental results at 7 and $8 \mathrm{TeV}$ ever.

At $13 \mathrm{TeV}$, top quark $p_{\mathrm{T}}$ spectra will be thoroughly investigated as never before, in a much extended range, in the LHC combinations of measurements in both resolved and boosted regimes. Any statistically limited measurement involving top quarks can profit from the combination of two independent datasets, specially during the first periods of Run II data taking. Examples of low statistics processes of major interest, that we suggest as potential candidates for LHCTOPWC combinations, are: $\mathrm{t} \overline{\mathrm{t}} \mathrm{b}, \mathrm{t} \overline{\mathrm{t}} \mathrm{V}$ (with $\mathrm{V}=\mathrm{W}, \mathrm{Z}, \gamma$ ), important backgrounds for $\mathrm{t} \overline{\mathrm{t}} \mathrm{H}$ production; flavour changing neutral current processes; single top and single anti-top quark production separately as probe of the proton PDF; spin correlations; ratio of branching fractions $R_{b}$; and others.

So far, only the ATLAS and CMS Collaborations at the LHC have been involved in the LHCTOPWG. However, very recently the LHCb Collaboration has observed top quark production in the forward for the first time [11].

In conclusion, through the intensive work carried out by experimentalists and theorists, and reflected on the combinations presented in this contribution, the LHC data is exploited to the fullest in terms of statistical precision, understanding (thus reduction) of the systematic uncertainties and theoretical scrutiny. That is one way of maximizing the LHC potential to unveil the last issues of the standard model to be understood, as well as to detect signs of new physics, if they are at all present at the LHC energies.

\section{References}

[1] Theory developments in differential top-quark distributions, D. Heymes, these proceedings.

[2] Statistical and systematic treatment issues in top mass combinations, A. Maier, these proceedings.

[3] How to combine correlated estimates of a single physical quantity, L. Lyons, D. Gibaut and P. Clifford, Nucl. Instrum. Meth. A 270, 110 (1988); Combining correlated measurements of several different physical quantities, A. Valassi, Nucl. Instrum. Meth. A, 500, 391 (2003). 
[4] Jet energy scale uncertainty correlations between ATLAS and CMS, ATLAS and CMS Collaborations, CMS PAS JME-14-004, ATL-PHYS-PUB-2014-020 (2014).

[5] Combination of ATLAS and CMS top quark pair cross section measurements in the e $\mu$ final state using proton-proton collisions at $\sqrt{s}=8 \mathrm{TeV}$, ATLAS and CMS Collaborations, CMS TOP-14-016, ATLAS-CONF-2014-054 (2014).

[6] Combination of ATLAS and CMS top-quark pair cross-section measurements using proton-proton collisions at $\sqrt{s}=7 \mathrm{TeV}$, ATLAS and CMS Collaborations, CMS TOP-12-003, ATLAS-CONF-2012-134 (2012).

[7] Combination of cross-section measurements of associated production of a single top-quark and a W boson at $\sqrt{s}=8 \mathrm{TeV}$ with the ATLAS and CMS experiments, ATLAS and CMS Collaborations, CMS TOP-14-009, ATLAS-CONF-2014-052 (2014).

[8] Combination of single top-quark cross-sections measurements in the t-channel at $\sqrt{s}=8 \mathrm{TeV}$ with the ATLAS and CMS experiments, ATLAS and CMS Collaborations, CMS TOP-12-002, ATLAS-CONF-2013-061 (2013).

[9] Combination of ATLAS and CMS t⿱亠t charge asymmetry measurements using LHC proton-proton collisions at $\sqrt{s}=7 \mathrm{TeV}$, ATLAS and CMS Collaborations, CMS TOP-14-006, ATLAS-CONF-2014-012 (2014).

[10] Combination of the ATLAS and CMS measurements of the W-boson polarization in top-quark decays, ATLAS and CMS Collaborations, CMS TOP-12-025, ATLAS-CONF-2013-033 (2013).

[11] First observation of top quark production in the forward region, LHCb collaboration, Phys. Rev. Lett. 115,112001 (2015). 